\title{
Oral Tolerance Induction by Bothrops jararaca Venom in a Murine Model and Cross-Reactivity with Toxins of Other Snake Venoms
}

\author{
Lilian Rumi Tsuruta $^{1, *(\mathbb{D})}$, Ana Maria Moro ${ }^{1} \mathbb{D}$, Denise V. Tambourgi ${ }^{2} \mathbb{D}$ and Osvaldo Augusto Sant'Anna ${ }^{2} \mathbb{D}$ \\ 1 Biopharmaceuticals Laboratory, Butantan Institute, São Paulo 05503-900, Brazil; ana.moro@butantan.gov.br \\ 2 Immunochemistry Laboratory, Butantan Institute, São Paulo 05503-900, Brazil; \\ denise.tambourgi@butantan.gov.br (D.V.T.); osvaldo.santanna@butantan.gov.br (O.A.S.) \\ * Correspondence: lilian.tsuruta@butantan.gov.br
}

Citation: Tsuruta, L.R.; Moro, A.M.; Tambourgi, D.V.; Sant'Anna, O.A. Oral Tolerance Induction by Bothrops jararaca Venom in a Murine Model and Cross-Reactivity with Toxins of Other Snake Venoms. Toxins 2021, 13, 865. https://doi.org/10.3390/ toxins 13120865

Received: 15 October 2021

Accepted: 30 November 2021

Published: 3 December 2021

Publisher's Note: MDPI stays neutral with regard to jurisdictional claims in published maps and institutional affiliations.

Copyright: (c) 2021 by the authors. Licensee MDPI, Basel, Switzerland. This article is an open access article distributed under the terms and conditions of the Creative Commons Attribution (CC BY) license (https:/ / creativecommons.org/licenses/by/ $4.0 /)$.

\begin{abstract}
Oral tolerance is defined as a specific suppression of cellular and humoral immune responses to a particular antigen through prior oral administration of an antigen. It has unique immunological importance since it is a natural and continuous event driven by external antigens. It is characterized by low levels of IgG in the serum of animals after immunization with the antigen. There is no report of induction of oral tolerance to Bothrops jararaca venom. Here, we induced oral tolerance to $B$. jararaca venom in BALB/c mice and evaluated the specific tolerance and cross-reactivity with the toxins of other Bothrops species after immunization with the snake venoms adsorbed to/encapsulated in nanostructured SBA-15 silica. Animals that received a high dose of B. jararaca venom (1.8 mg) orally responded by showing antibody titers similar to those of immunized animals. On the other hand, mice tolerized orally with three doses of $1 \mu \mathrm{g}$ of $B$. jararaca venom showed low antibody titers. In animals that received a low dose of $B$. jararaca venom and were immunized with $B$. atrox or B. jararacussu venom, tolerance was null or only partial. Immunoblot analysis against the venom of different Bothrops species provided details about the main tolerogenic epitopes and clearly showed a difference compared to antiserum of immunized animals.
\end{abstract}

Keywords: oral tolerance; Bothrops jararaca; snake venom; ELISA

Key Contribution: We describe the induction of oral tolerance by administration of Bothrops jararaca venom through an original approach, showing the main tolerogenic epitopes in B. jararaca venom and other species of Bothrops.

\section{Introduction}

Snake venoms are composed of a high diversity of proteins and peptides with biological activities, allowing these animals to defend themselves and immobilize their prey [1]. The composition of snake venoms among species displays high variability, both in qualitative and quantitative aspects and complexity [2]. Accidents with snakebite envenoming cause local and systemic effects and represent a public health problem in developing countries, where they reach lower socio-economic segments and kill $>100,000$ people each year [3]. The primary treatment for the systemic effects of snake envenoming is the intravenous administration of antivenom against specific venoms. Antivenoms specifically neutralize the venoms used in their production and those of related species, which means that antivenoms are produced regionally depending on demand [3]. Indeed, there is a crisis related to the supply of antivenoms, especially in sub-Saharan Africa and parts of Asia; the development of new treatments for patients with snakebite envenoming should be promoted on the basis of recent scientific knowledge related to snake venoms [3]. Recently, several studies have reported that antivenom serum antibodies, generated against specific snake venoms, are cross-reactive with venoms from other species, considering homologous and heterologous snake venoms [4-7]. 
Most snakebites in Brazil occur because of the genus Bothrops and are considered a serious public health problem. Bothrops venom components mainly cause local damage and systemic effects targeting blood hemostasis, endothelial microcirculation, extracellular matrix, and the cardiovascular system [1,8].

Oral tolerance is the induction of peripheral immune tolerance by the oral administration of the antigen and is characterized by the inhibition of the specific immune response to this antigen due to prior oral exposure [9-14]. It is a natural and continuous process driven by external antigens. It has a unique immunological importance, as it develops unresponsiveness to ingested food and potential insults from the environment to maintain host homeostasis by protecting against food allergies and colitis caused by autoimmunity $[12,13,15]$. The gut is regularly exposed to multiple types of antigens, and the associated immune system has specialized immune cells and lymph nodes to balance responses to commensal bacteria (microbiome), innocuous antigens, and harmful microorganisms [11]. Depending on the properties of the antigen, such as size and solubility, the orally administrated antigen that reaches the intestinal epithelium is transported by different routes and can lead to the induction of tolerance or immunity [14]. The oral tolerance induction mechanism has been extensively studied using animal models, mainly for food allergens [11]. It involves multiple factors, and it is known that the dose of the administered antigen and the consumption time are decisive. Administration of a single high dose of antigen leads to the mechanisms of anergy or depletion, whereas exposure to multiple low doses favors the development of regulatory T cells [11,16]. Anergy induction means obtaining antigen-unresponsive T cells, while depletion induction refers to apoptosis of antigen-specific T cells [14]. Previous studies have shown that genetic and environmental factors are involved in the induction of oral tolerance, demonstrating that this characteristic is a process under the influence of multiple factors [17-19].

Oral tolerance induction by the administration of one kind of antigen/allergen has been extensively investigated, as has been the mechanism of this process involving immune cells and pathways $[11,13,20]$. This process has not been explored by the administration of a complex mixture of proteins. Snake envenomation by the oral route does not occur in nature; instead, snakes inject their venoms when there is a dangerous situation and/or they need to defend themselves. Oral antigen application of this kind to induce oral tolerance represents a novel experimental approach. To our knowledge, there is no report of oral tolerance induction using $B$. jararaca venom as an antigen. We propose a method for inducing oral tolerance to B. jararaca venom in mice, followed by evaluation of serum crossreactivity with the toxins from other Bothrops species compared to the serum generated by the immunization process.

\section{Results}

\subsection{Induction of Oral Tolerance by B. jararaca Venom Is Dependent on Dose Administed}

Oral tolerance is characterized by low IgG levels in the antiserum of animals after immunization with the antigen previously administered orally. Initially, the induction of oral tolerance by administering $B$. jararaca venom was tested using two conditions and the results are summarized in Figure 1. Group I did not receive venom orally. Briefly, one group of mice orally received a single dose of $1.8 \mathrm{mg}$ of the venom (Group II) and other groups received $1 \mu \mathrm{g}$ of snake venom on the first, third and fifth day (Groups III and IV). At 12 days after the last low dose of $1 \mu \mathrm{g}$, all groups were immunized with snake venom combined with silica adjuvant. Groups I, II and III received venom from B. jararaca, while Group IV was challenged with venom from B. atrox. Antisera corresponding to 7 days after immunization were analyzed, and no difference in antibody titers between all groups of mice was observed (data not shown). Antisera were then collected on the 35th day after immunization and titers determined (Figure 1), followed by analysis based on ANOVA. According to our results, animals that received high doses of B. jararaca venom orally were not tolerized (Group II), with antibody titers being similar to those of mice immunized only (Group I). No statistically significant differences were found between Groups I and II 
and Groups I and IV. Interestingly, mice given a low dose of venom orally and immunized with the same antigen (Group III) had the lowest antibody titer showing the induction of oral tolerance with a statistically significant difference compared to other groups. On the other hand, animals that received a low oral dose of B. jararaca and were immunized with B. atrox venom (Group IV) showed no tolerance induction. Our results suggest that oral tolerance to snake venom appears to be dose-dependent and is specific for the venom of each snake species.

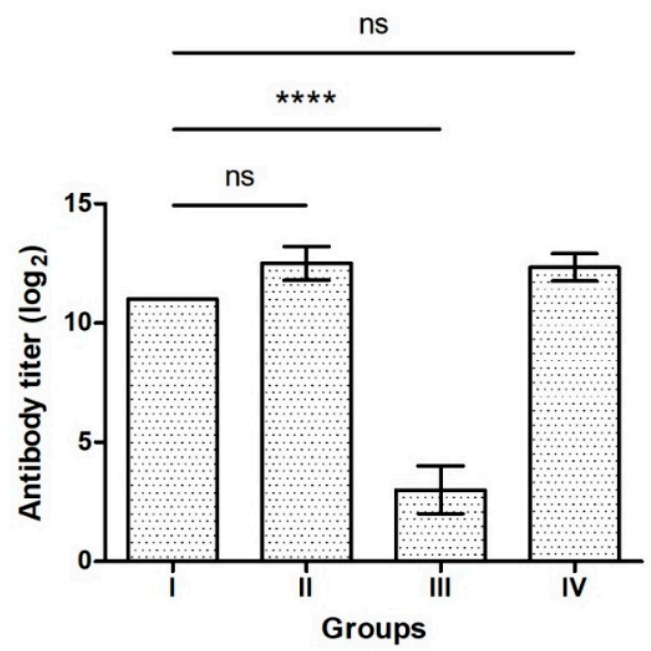

Group I: mice immunized with $1 \mu \mathrm{g}$ of $B$. jararaca venom

Group II: mice orally tolerized with $1.8 \mathrm{mg}$ of $B$. jararaca venom and immunized with $1 \mu \mathrm{g}$ of $B$. jararaca venom Group III: mice orally tolerized with 3 doses of $1 \mu \mathrm{g}$ of $B$. jararaca venom and immunized with $1 \mu \mathrm{g}$ of $B$. jararaca venom Group IV: mice orally tolerized with 3 doses of $1 \mu \mathrm{g}$ of $B$. jararaca and immunized with $1 \mu \mathrm{g}$ of $B$. atrox venom

Figure 1. Induction of oral tolerance by administration of $B$. jararaca venom evaluated by antibody titer of mouse antiserum after immunization. Group I was not subjected to oral tolerance. The mice were orally tolerized with a single high dose of venom (Group II) or with low doses of venom on the first, third and fifth day (Group III and IV). Twelve days after receiving the last oral dose, Groups I, II and III were immunized with B. jararaca venom, and group IV received B. atrox venom. Serum antibody titers corresponding to 35 days after immunization were determined by ELISA. Error bars represent the standard deviation of an experiment $(n=3)$. ANOVA with $95 \%$ confidence intervals was used to determine significant differences between each group of mice. ns: not significant; ****: $p$-value was $<0.0001$.

\subsection{Characterization of Antisera from Orally Tolerized Animals Reveals Specificity for Other Snake Venoms}

To characterize the specificity and cross-reactivity of the antiserum after induction of oral tolerance with $B$. jararaca venom, another experiment was carried out with induction of oral tolerance by administering three doses of $1 \mu \mathrm{g}$ of the B. jararaca venom (Groups I and III). Eight days after the last dose, all groups of mice were immunized intraperitoneally with snake venom combined to adjuvant. Groups I and II were immunized with B. jararaca, while Groups III and IV received B. jararacussu venom. Antiserum was collected 12, 25 and 45 days after immunization to analyze the specificity of response to $B$. jararaca and $B$. jararacussu venoms. The specificity of antiserum for the venom of $B$. jararaca, using this venom as the antigen is shown in Figure 2, while Figure 3 shows the specificity for the venom of B. jararacussu. Our results showed that Group I, orally tolerized and immunized with $B$. jararaca venom, was tolerized for snake venom in view of the low IgG level in all analyzed antisera (Figures 2A and 3A). Group III was tolerized orally and immunized with $B$. jararacussu venom and the antibody titer increased on the 45th day after venom injection when specificity for B. jararaca venom was evaluated (Figure 2C), while the same phenomenon was observed on day 25 for the specificity of B. jararacussu venom (Figure 3C). Antiserum from groups of mice immunized with snake venom only (Groups II and IV) had high antibody titers for both snake venoms 25 days after immunization 
(Figures 2B,D and 3B,D). Furthermore, antisera from mice immunized with $B$. jararacussu venom (Group IV) showed reactivity with $B$. jararaca venom (Figure $2 B$ ) and antisera from mice immunized with $B$. jararaca venom (Group II) showed reactivity with $B$. jararacussu venom (Figure 3B). In the case of specificity for B. jararacussu venom, mice immunized with B. jararaca venom (Group II) showed a reduction in antibody titer 45 days after immunization (Figure 3B).

\section{A}

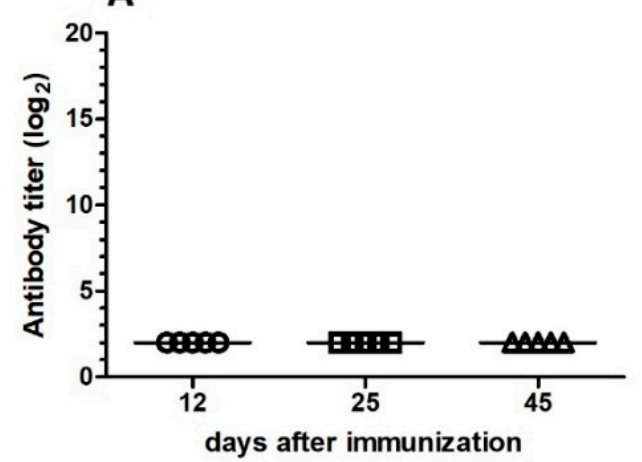

C

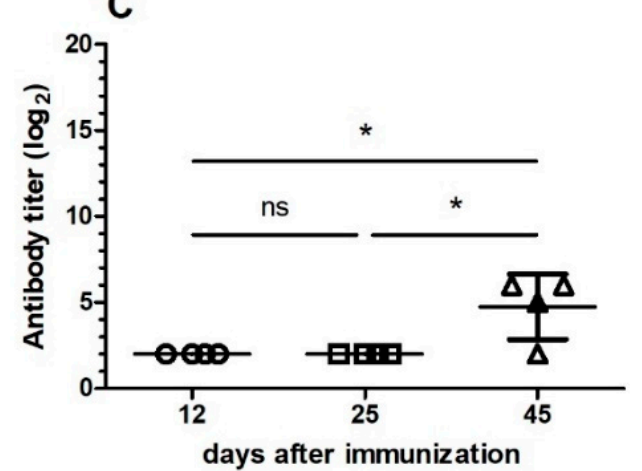

B
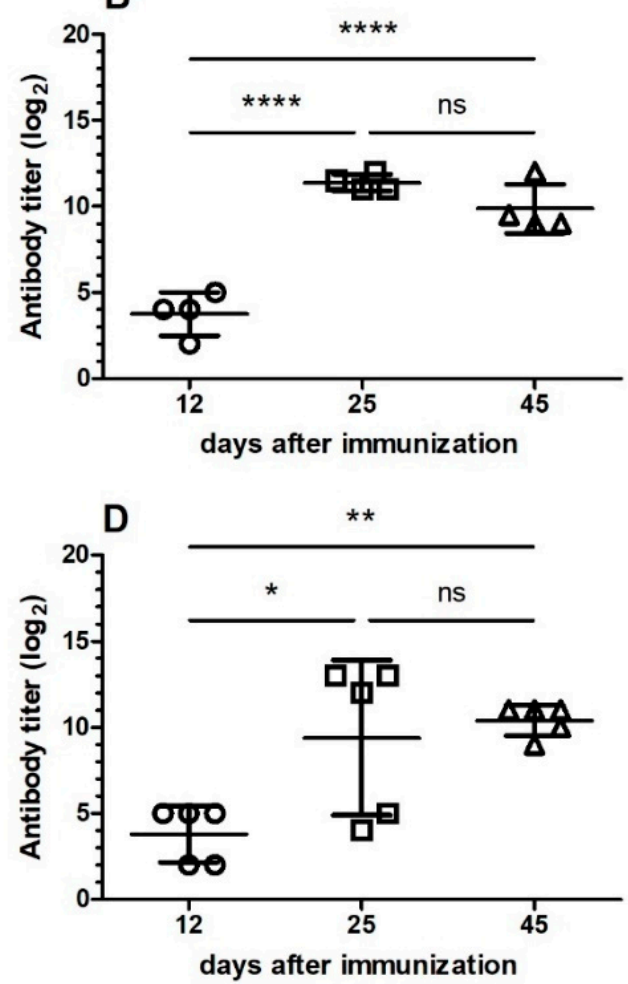

Figure 2. Antiserum specificity for B. jararaca venom from mice subjected or not to oral tolerance followed by immunization with snake venom. Microplate wells were coated with $1 \mu \mathrm{g}$ of $B$. jararaca venom, and serial dilutions of antiserum of Groups I-IV corresponding to 12, 25 and 45 days after snake venom immunization were applied; antibody titer was determined by ELISA. (A) Group I: mice tolerized with $B$. jararaca venom and immunized with the same snake venom; (B) Group II: mice immunized with $B$. jararaca venom; (C) Group III: mice tolerized with B. jararaca venom and immunized with B. jararacussu venom; (D) Group IV: mice immunized with B. jararacussu venom. The mean and standard deviation $(n=5)$ of each antibody titer obtained were plotted. ANOVA with $95 \%$ confidence intervals was used to determine significant differences between each group of mice group. ns: not significant; ${ }^{*}: p$-value was $0.01-0.05 ;^{* *}$ : $p$-value was $0.001-0.01 ;{ }^{* * * *}: p$-value was $<0.0001$.

IgG1 and IgG2a titers were determined for antisera obtained 45 days after immunization for Groups I, II, III and IV (Figure 4). Orally tolerized mice had lower antibody titers than immunized mice and no difference between IgG1 and IgG2a titers was observed. $\operatorname{IgG1}$ antibody titers from mice immunized with snake venoms (B. jararaca or B. jararacussu venom) were higher than IgG2a titers. IgE was not found in any of the four groups (data not shown). 
A

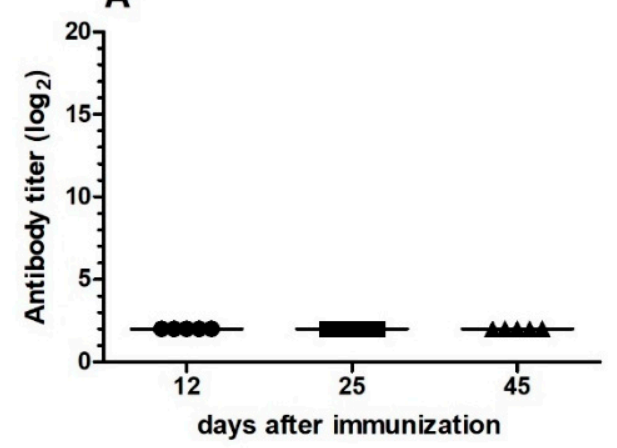

C

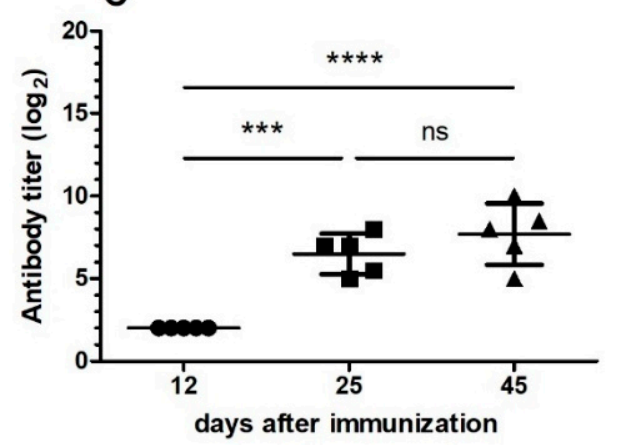

B
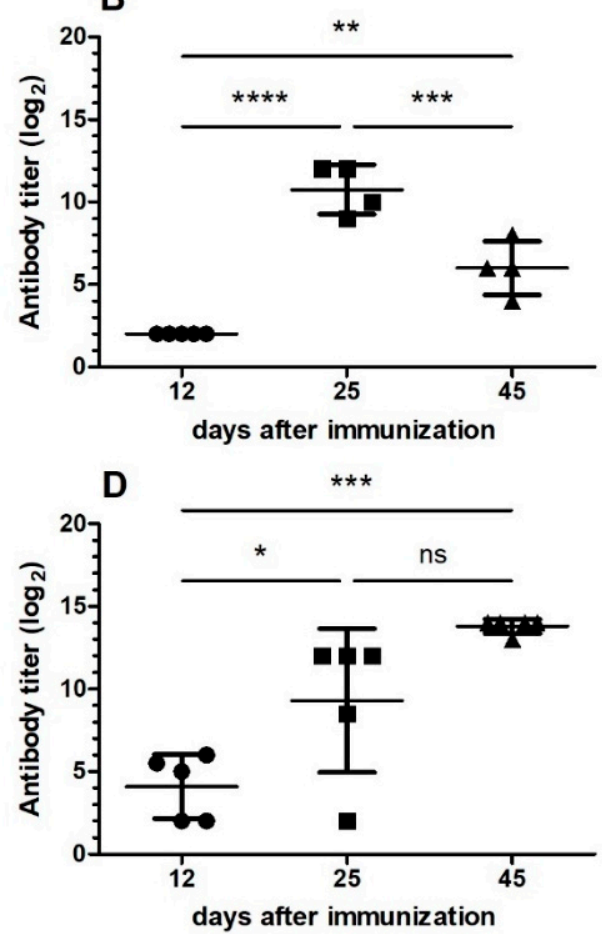

Figure 3. Antiserum specificity for B. jararacussu venom from mice subjected or not to oral tolerance followed by immunization with snake venom. Microplate wells were coated with $1 \mu \mathrm{g}$ of B. jararacussu venom, and serial dilutions of antiserum of Groups I-IV corresponding to 12, 25 and 45 days after snake venom immunization were applied; antibody titer was determined by ELISA. (A) Group I: mice tolerized with B. jararaca venom and immunized with the same snake venom; (B) Group II: mice immunized with B. jararaca venom; (C) Group III: mice tolerized with B. jararaca venom and immunized with B. jararacussu venom; (D) Group IV: mice immunized with B. jararacussu venom. The mean and standard deviation $(n=5)$ of each antibody titer obtained were plotted. ANOVA with $95 \%$ confidence intervals was used to determine significant differences between each mice group. ns: not significant; *: $p$-value was $0.01-0.05 ;{ }^{* *}$ : $p$-value was $0.001-0.01 ;{ }^{* * *}: p$-value was $0.0001-0.001 ;{ }^{* * * *}: p$-value was $<0.0001$.

Next, we evaluated the reactivity of antisera from mice subjected to oral tolerance with venoms of some Bothrops species and Bitis anetans by Western blotting. Protein profiles of the snake venoms under non-reducing conditions are shown in Figure 5; venoms differed in composition and band intensity.

Snake venoms subjected to electrophoresis were transferred to PVDF membranes, the membrane was incubated with a pool of antisera corresponding to 45 days after the immunization of Groups I, II, III and IV and revealed by chemiluminescent reagent (Figure 6). Antisera from orally tolerized animals showed lower reactivity with snake venom proteins compared to antisera obtained from mice immunized with snake venom. Antisera of mice tolerized with $B$. jararaca venom and immunized with the same snake venom (Group I) recognized some proteins from the venom of $B$. jararaca, and a protein band from the venom of B. alternatus, B. jararacussu and B. atrox amazonia (Figure 6A). On the other hand, the antisera from mice immunized with the venom of $B$. jararaca recognized venom proteins of the Bothrops species analyzed (Bothrops jararaca, B. alternatus, B. jararacussu and $B$. atrox amazonia), and a wide range of reactivity was found for the venoms of $B$. jararaca and B. alternatus (Figure 6B). Interestingly, some protein bands recognized by both tolerized and non-tolerized mouse antisera (Groups I and II) were very similar (Figure 6A,B). When antisera from mice orally tolerized or not and immunized with $B$. jararacussu venom (Groups III and IV) were evaluated, at least one protein of the snake venom was recognized by antisera from Group III (Figure 6C), and antisera from immunized mice (Group IV) 
showed higher reactivity with $B$. jararacussu and B. jararaca venoms and low reactivity with other snake venoms (Figure 6D). The reactivity of Groups III and IV antisera was tested for Bitis anetans venom, and antisera from tolerized or not mice showed reactivity with this venom. Each antiserum recognized a distinct protein (Figure 6C,D). Our results showed that antisera from mice tolerized orally with $B$. jararaca venom and immunized with snake venoms had reactivity with venoms from other species at different levels. Interestingly, antisera from orally tolerized mice demonstrated cross-reactivity with different venom epitopes in comparison to the antisera of immunized animals.
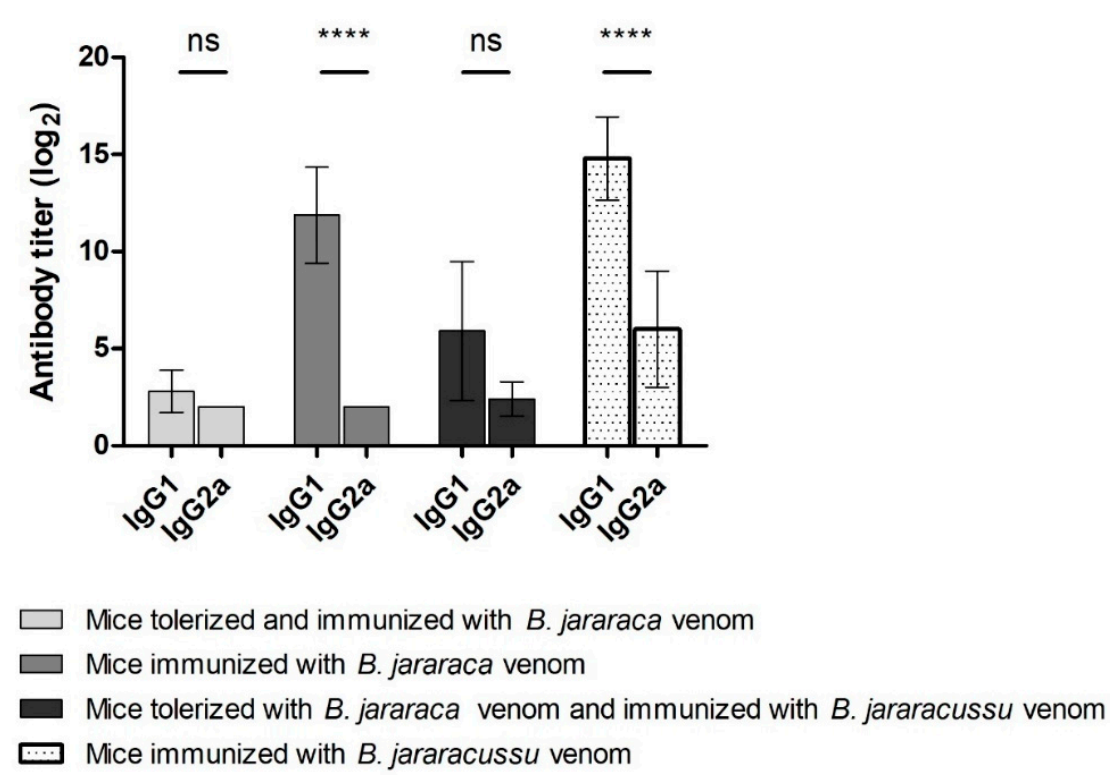

Figure 4. IgG1 and IgG2a antibody titers of groups of mice subjected to oral tolerance induction with B. jararaca venom followed by immunization. Microplate wells were coated with $1 \mu \mathrm{g}$ of $B$. jararaca venom, and serial dilutions of Groups I-IV antiserum, as previously described in "Section 4", corresponding to 45 days after immunization with snake venom, were applied; antibody titer was determined by ELISA using mouse anti-IgG1 or anti-IgG2a antibodies conjugated to HRP. Error bars represent the standard deviation of one experiment $(n=5)$. 2-way ANOVA with $95 \%$ confidence intervals was used to determine significant difference between IgG1 and IgG2a antibody titers for each group. ns: not signigficant; ${ }^{* * *}:$-value was $<0.0001$.

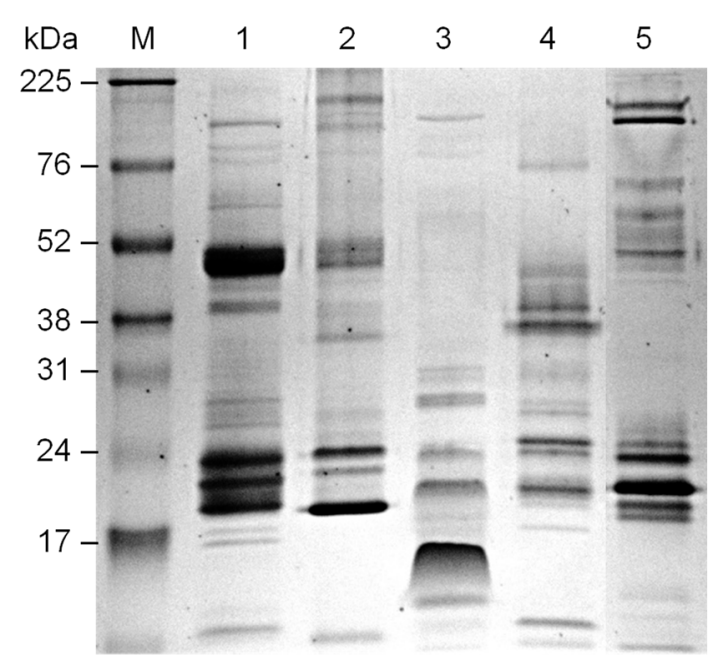

Figure 5. Electrophoretic profiles of snake venoms under non-reducing conditions. 12\% SDS-PAGE of $5 \mu \mathrm{g}$ of each snake venom. Coomassie blue staining. M: Rainbow marker high range (Cytiva); snake venom from 1: B. jararaca; 2: B. alternatus; 3: B. jararacussu; 4: B. atrox amazonia; 5: Bitis anetans. 


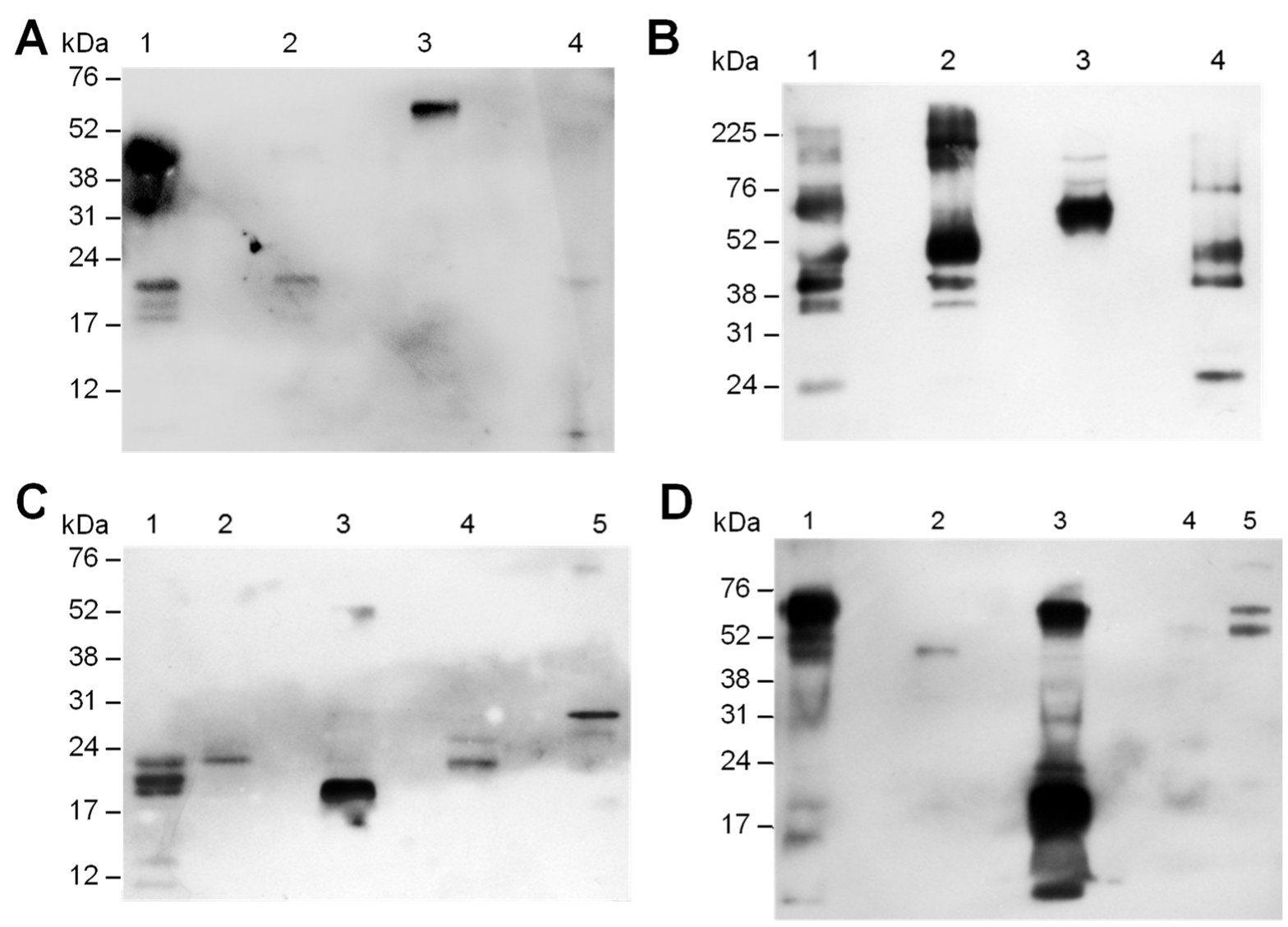

Figure 6. Analysis of the reactivity of the pool of antisera from animals subjected or not to oral tolerance induction with B. jararaca venom followed by immunization with snake venom. Snake venoms were subjected to electrophoresis and transferred to PVDF membrane according to Figure 5. The membrane was incubated with a pool of antisera corresponding to 45 days after immunization. (A): 1/400 dilution of Group I antisera; (B): 1/1000 dilution of Group II antisera; (C): 1/400 dilution of Group III antisera; (D) 1/1500 dilution of Group IV antisera. Peroxidase-conjugated anti-mouse IgG was then added, and the membrane was revealed with ECL reagent.

\section{Discussion}

Oral tolerance is an immunological process in which the specific immune response is inhibited by prior oral administration of antigen. The induction of this process can be assessed after antigen immunization and measured by determination of antibody titer or by the decrease in allergic symptoms after allergen challenge [14].

The ability of the immune system to adapt to dietary antigens and commensal bacteria is important and prevents the development of food allergies, celiac disease, and autoimmune diseases [20]. Most studies related to oral tolerance have been carried out in animal models to establish the safe dose and duration of the process and to understand the immune cells and pathways involved, because of the risk of testing in humans [20]. These studies are of crucial importance for understanding the mechanisms involved and can promote strategies for the development of natural oral tolerance and prevent intoxication, allergies, and autoimmune diseases.

There is a report of daily oral administration for 14 days of Crotalus durissus terrificus snake venom $(200 \mu \mathrm{g} / \mathrm{kg})$ in male Swiss mice (17-21 g) that induced tolerance to the antinociceptive effect, and no antibody titers were measurable after prolonged treatment [21]. The expected effect was obtained by administering a dose of venom higher than that in our study, and mice were not immunized after receiving the oral dose. The cited study had a different purpose in relation to the present work and a different approach was taken. 
Most incidences of snakebites in Brazil, considering all regions, are related to Bothrops species [22] and, therefore, constituted the objective of this study. They are also extensively investigated because they are responsible for more fatality cases in Central and South America than other groups of snakes [1]. So far, oral tolerance by administering B. jararaca venom in animal models has not been published and we have successfully established a protocol in BALB/c mice. The antibody titers were measured in the antisera after immunization with snake venoms to understand the mechanism involved.

We observed that mice receiving a single dose of $1.8 \mathrm{mg}$ of $B$. jararaca venom orally did not develop tolerance and that the antibody titer was similar to that of the group of mice that was only immunized (Figure 1). These results are in agreement with those found in the literature for other antigens $[11,16]$. We have shown that repeated exposure to a dose of $1 \mu \mathrm{g}$ of $B$. jararaca venom induced tolerance (Figure 1). We also found that tolerance was more effectively induced when animals received the same snake venom during oral administration and immunization (Figures 1-3), showing that it is specific for one type of antigen, even when the antiserum showed cross-reactivity with venom from other snakes.

There was no reference concerning an immunization protocol with snake venom after oral administration, and we established that immunization would be performed about 7 days after the last dose. In the first protocol, mice were immunized 12 days after the last dose, while in the second protocol it was 8 days, and under both conditions, the oral tolerance induction was verified by low antibody titer. We think that the immunization protocol could be applied in this period of time to induce oral tolerance with $B$. jararaca venom.

In the first protocol of oral tolerance induction, mice received a low dose of B. jararaca venom and were immunized with $B$. atrox venom, and no tolerance induction was observed (Figure 1). On the basis of this result, we excluded this condition in the next protocol of oral tolerance induction and challenged the immunization with the venom of another Bothrops species (B. jararacussu). Partial oral tolerance induction was observed (Figures 2 and 3) with B. jararacussu venom immunization, showing that this tolerance can be induced with another Bothrops venom. Variations in venom composition and biological activities of Brazilian snakes from Bothrops genus were observed [23] and these differences could be influenced on oral tolerance induction when immunization with heterologous venom was applied. Further studies involving different conditions for establishing oral tolerance induction with $B$. jararaca venom and other Bothrops species could elucidate these observations.

We also performed immunoblot analysis of the venom of different species of Bothrops incubating with antisera from animals tolerized with administration of $B$. jararaca venom or only immunized with snake venom. The epitopes recognized by each antiserum were clearly different (Figure 6), showing that the reactivity profile of antisera in relation to venom components changed according to the protocol used to induce tolerance. To see if proteins in snake venom from species other than Bothrops are recognized by antisera from mice orally tolerized or not, reactivity with Bitis anetans venom was evaluated. Antisera from mice partially tolerized (Group III) and from animals immunized with B. jararacussu venom (Group IV) were tested and each antiserum recognized different proteins in Bitis anetans venom, showing that reactivity with other snake venoms could be explored in future studies.

The application of oral tolerance has advantages because it is non invasive and uses a simple route. New knowledge and in-depth understanding of this process could contribute to the application of oral tolerance in prophylaxis and treatment of diseases [20].

Most of the studies related to oral tolerance induction involve the administration of one antigen $[11,13,20]$. The present work is an experimental study of oral tolerance induction developed with $B$. jararaca venom that is composed of a complex of proteins with biological activity. This process does not happen in the nature, and thus, the observation of oral tolerance induction represented a novel experimental approach. To demonstrate oral tolerance induction in our approach, the immune response was evaluated by the determination of the antibody titer, and then, the epitopes recognized by antiserum from 
orally tolerized or not tolerized mice were compared. Our results showed that it is possible to induce oral tolerance by administration of the complex mixture of the proteins, such as $B$. jararaca venom. Investigations related to the modulation of immune response and the mechanisms involved in this process were not part of the scope of the present work. The administration of a single high dose of $B$. jararaca venom was not lethal to the animals but did not induce oral tolerance. Further experimental studies should be conducted with other snake venoms to confirm this phenomenon as this approach would be used to develop vaccines by oral administration. The protocol established for oral tolerance induction should also be applied to other snake venoms in future studies together with the investigation of the mechanisms involved in this process. Therefore, the present work opened new perspectives to explore the process of oral tolerance induction.

\section{Materials and Methods}

\subsection{Animals}

BALB/c mice, female, aged 2-4 months, were used and maintained at the animal facilities of the Immunochemistry Laboratory, Butantan Institute, and they were caged and handled according to the International Animal Welfare Recommendations and in line with the guidelines for the use of animals in biomedical research [24]. Ethics Committee on Animal Use of the Butantan Institute approved the experiment protocol (Protocol IBU 454/08; 9 April 2008).

\subsection{Snake Venoms}

Lyophilized venoms (Bothrops jararaca, B. alternatus, B. jararacussu, B. atrox amazonia and Bitis anetans) were obtained from the Laboratory of Herpetology, Butantan Institute, São Paulo, Brazil, and stored at $-20^{\circ} \mathrm{C}$. Venoms were resuspended in phosphate-buffered saline (PBS).

\subsection{Protein Quantification}

Protein concentration of the snake venom samples was determined by a microtiterbased Bradford Protein Assay (BioRad) in microplates using bovine serum albumin (BSA-Sigma-Aldrich, St. Louis, MO, USA) for the standard curve [25].

\subsection{Induction of Oral Tolerance and Immunization}

To establish the oral tolerance induction protocol, BALB/c mice were subdivided into 4 groups of animals $(n=3)$. Group I did not receive venom orally. Oral tolerance was induced by gavage administration of B. jararaca venom, and the mice received a single high dose of $1.8 \mathrm{mg}$ (Group II) or 3 doses of $1 \mu \mathrm{g}$ on the first, third and fifth day (Groups III and IV). At 12 days after the last low dose (Groups III and IV), all groups were intraperitoneally immunized with $1 \mu \mathrm{g}$ of snake venom adsorbed to/encapsulated in nanostructured SBA-15 silica [26]. Groups I, II and III received B. jararaca venom, while Group IV was immunized with $B$. atrox venom. Antiserum corresponding to the 7th and 35th day after immunization was collected by bleeding from the retroorbital plexus and the antibody titer was determined by ELISA. Animals that did not undergo any procedure $(n=3)$ were considered the control group, and sera were added to the antibody titer assay.

Another oral tolerance induction protocol was then carried out. BALB/c mice were subdivided into 4 groups of animals $(n=5)$. Oral tolerance was induced by gavage administration of $1 \mu \mathrm{g}$ of the B. jararaca venom on the first, third and fifth day (Groups I and III). Groups II and IV did not receive venom orally. Eight days after the last dose, mice were intraperitoneally immunized with $1 \mu \mathrm{g}$ of snake venom adsorbed to/encapsulated in nanostructured SBA-15 silica. Groups I and II were immunized with B. jararaca, while Groups III and IV received B. jararacussu venom. Antiserum was collected by bleeding from the retroorbital plexus at 12, 25 and 45 days after immunization, and the antibody titers were determined by ELISA. Sera from mice of control group were also added. The cross- 
reactivity of serum after induction of oral tolerance with $B$. jararaca venom was analyzed by Western blotting.

\subsection{ELISA Procedure}

IgG titer of mouse sera was determined by ELISA. B. jararaca or B. jararacussu venom was diluted to $10 \mu \mathrm{g} / \mathrm{mL}$ in carbonate buffer $\left(50 \mathrm{mM} \mathrm{Na} 2 \mathrm{CO}_{3} / \mathrm{NaHCO}_{3}, \mathrm{pH} 9.6\right)$, and $100 \mu \mathrm{L} /$ well were added to a 96-well EIA/RIA Clear Flat Bottom Polystyrene High Bind Microplate (3590, Corning, NY, USA). The plate was incubated at $4{ }^{\circ} \mathrm{C}$ overnight, washed 3 times with PBS $/ 0.05 \%$ Tween and then incubated with the PBS/5\% BSA blocking solution at $37^{\circ} \mathrm{C}$ for $2 \mathrm{~h}$. The blocking solution was replaced with a serial twofold dilution of antiserum in PBS/1\% BSA, starting at an appropriate dilution for each one. Sera from animals that did not receive any venom was used as negative control, and samples were incubated at $37^{\circ} \mathrm{C}$ for $1 \mathrm{~h}$. Wells were washed 5 times with PBS $/ 0.05 \%$ Tween to remove unbound antibodies. HRP-conjugated anti-mouse IgG $(\mathrm{H}+\mathrm{L})$ (Promega, Madison, WI, USA) was used as secondary antibody at $1 / 2500$ dilution, and incubation was at $37^{\circ} \mathrm{C}$ for $1 \mathrm{~h}$. The plate was washed again, and the substrate used was OPD (o-phenylenediamine) (SigmaAldrich) in phosphate-citrate buffer with $\mathrm{H}_{2} \mathrm{O}_{2}$. After 5 min incubation, the reaction was stopped by adding $0.2 \mathrm{M}$ citric acid. The absorbance at $450 \mathrm{~nm}$ was measured with a microplate reader. Antibody titers were expressed as $\log 2$ maximum serum dilution giving a positive reaction at which the absorbance was equal to two times the control value of the control serum.

Immunoglobulin isotypes, IgG1 and IgG2a, were evaluated in mouse antisera. For this purpose, HRP rat anti-mouse IgG1 (BD Biosciences, San Jose, CA, USA) at 1/400 dilution and HRP rat anti-mouse IgG2a (BD Biosciences) at 1/1000 dilution were used as secondary antibody. Antibody titer was determined as described above.

\subsection{Electrophoresis and Western Blotting}

Venom samples of $5 \mu \mathrm{g}$ were prepared by adding non-reducing sample buffer and were separated by electrophoresis on 12\% SDS-PAGE gel. Amersham ECL High-Range Rainbow Molecular Weight Markers (Cytiva, Marlborough, MA, USA) was also included. The gel was stained with Coomassie Blue or transferred to Amersham Hybond-P PVDF Membrane (Cytiva) for $1 \mathrm{~h}$ at $10 \mathrm{~V}$ using a BioRad Trans-blot SD semi-dry transfer cell. The membranes were stained with $0.5 \%$ Ponceau S, washed with ultrapure water and then blocked with PBS $/ 5 \%$ BSA at $37^{\circ} \mathrm{C}$ for $2 \mathrm{~h}$. Next, the membranes were washed twice with PBS $/ 0.1 \%$ Tween 20 for $20 \mathrm{~s}$ and were incubated overnight with an appropriate dilution of pooled antisera (Groups I, II, III and IV corresponding to 45 days after immunization) at $4{ }^{\circ} \mathrm{C}$. The membranes were washed 4 times for $5 \mathrm{~min}$ and incubated with anti-mouse IgG (whole molecule)-HRP (Sigma) at 1/30,000 dilution in PBS/1\% BSA for $1 \mathrm{~h}$ at room temperature. After washing, the blots were developed using Amersham ECL Prime Western Blotting Detection Reagent (Cytiva).

\subsection{Statistical Analysis}

Statistical analysis was performed by GraphPad Prism software. ANOVA (Tukey's multiple comparison test) was used to compare average antibody titer obtained between antisera of animal groups, orally tolerized or not with $B$. jararaca venom. The sera obtained at different times after immunization were submitted to analyses. Two-way ANOVA with $95 \%$ confidence intervals was used to determine significant differences between IgG1 and IgG2a antibody titers for each mouse group.

Author Contributions: Conceptualization, O.A.S.; methodology, L.R.T. and O.A.S.; validation, L.R.T. and O.A.S.; formal analysis, L.R.T., D.V.T. and O.A.S.; investigation, L.R.T. and O.A.S.; resources, A.M.M., D.V.T. and O.A.S.; data curation, L.R.T.; writing —original draft preparation, L.R.T.; writingreview and editing, L.R.T., A.M.M. and D.V.T.; visualization, L.R.T., A.M.M., D.V.T. and O.A.S.; supervision, O.A.S.; project administration, O.A.S.; funding acquisition, A.M.M., D.V.T. and O.A.S. All authors have read and agreed to the published version of the manuscript. 
Funding: This research was funded by Brazilian National Council for Scientific and Technological Development (CNPq), grant number 474435/2007-5, by INCTTOX (Instituto Nacional de Ciência e Tecnologia em Toxinas) from the São Paulo Research Foundation (FAPESP) and CNPq, and by FAPESP (grant number 2017/17844-8). A.M.M. is grateful for CNPq (process 307405/2020-0).

Institutional Review Board Statement: The methodology of this work was approved by Ethic Committee on Animal Use of the Butantan Institute (Protocol IBU 454/08, 9 April 2008).

Informed Consent Statement: Not applicable.

Data Availability Statement: The data presented in this study are available on request from corresponding author.

Acknowledgments: We thank Stephanie Murari do Nascimento for technical support.

Conflicts of Interest: The authors declare no conflict of interest.

\section{References}

1. Tashima, A.K.; Zelanis, A.; Kitano, E.S.; Ianzer, D.; Melo, R.L.; Rioli, V.; Sant'anna, S.S.; Schenberg, A.C.; Camargo, A.C.; Serrano, S.M. Peptidomics of three Bothrops snake venoms: Insights into the molecular diversification of proteomes and peptidomes. Mol. Cell Proteom. 2012, 11, 1245-1262. [CrossRef]

2. Tasoulis, T.; Isbister, G.K. A Review and Database of Snake Venom Proteomes. Toxins 2017, 9, 290. [CrossRef]

3. Gutiérrez, J.M.; Calvete, J.J.; Habib, A.G.; Harrison, R.A.; Williams, D.; Warrell, D.A. Snakebite envenoming. Nat. Rev. Dis. Primers 2017, 3, 17063. [CrossRef]

4. Lipps, B.V.; Khan, A.A. Antigenic cross reactivity among the venoms and toxins from unrelated diverse sources. Toxicon 2000, 38, 973-980. [CrossRef]

5. Prieto da Silva, A.R.; Yamagushi, I.K.; Morais, J.F.; Higashi, H.G.; Raw, I.; Ho, P.L.; Oliveira, J.S. Cross reactivity of different specific Micrurus antivenom sera with homologous and heterologous snake venoms. Toxicon 2001, 39, 949-953. [CrossRef]

6. Stábeli, R.G.; Magalhães, L.M.; Selistre-de-Araujo, H.S.; Oliveira, E.B. Antibodies to a fragment of the Bothrops moojenil-amino acid oxidase cross-react with snake venom components unrelated to the parent protein. Toxicon 2005, 46, 308-317. [CrossRef] [PubMed]

7. Rocha, M.M.; Paixão-Cavalcante, D.; Tambourgi, D.V.; Furtado, M.F. Duvernoy's gland secretion of Philodryas olfersii and Philodryas patagoniensis (Colubridae): Neutralization of local and systemic effects by commercial bothropic antivenom (Bothrops genus). Toxicon 2006, 47, 95-103. [CrossRef] [PubMed]

8. Mamede, C.C.N.; Simamoto, B.B.S.; Pereira, D.F.C.; Costa, J.O.; Ribeiro, M.S.M.; de Oliveira, F. Edema, hyperalgesia and myonecrosis induced by Brazilian bothropic venoms: Overview of the last decade. Toxicon 2020, 187, 10-18. [CrossRef] [PubMed]

9. Faria, A.M.; Ficker, S.M.; Speziali, E.; Menezes, J.S.; Stransky, B.; Verdolin, B.A.; Lahmann, W.M.; Rodrigues, V.S.; Vaz, N.M. Aging and immunoglobulin isotype patterns in oral tolerance. Braz. J. Med. Biol. Res. 1998, 31, 35-48. [CrossRef]

10. Sun, J.B.; Czerkinsky, C.; Holmgren, J. Mucosally induced immunological tolerance, regulatory T cells and the adjuvant effect by cholera toxin B subunit. Scand. J. Immunol. 2010, 71, 1-11. [CrossRef] [PubMed]

11. Commins, S.P. Mechanisms of Oral Tolerance. Pediatr. Clin. N. Am. 2015, 62, 1523-1529. [CrossRef]

12. Anagnostou, K.; Clark, A. What do we mean by oral tolerance? Clin. Exp. Allergy 2016, 46, 782-784. [CrossRef]

13. Sricharunrat, T.; Pumirat, P.; Leaungwutiwong, P. Oral tolerance: Recent advances on mechanisms and potential applications. Asian Pac. J. Allergy Immunol. 2018, 36, 207-216. [CrossRef]

14. Tordesillas, L.; Berin, M.C. Mechanisms of Oral Tolerance. Clin. Rev. Allergy Immunol. 2018, 55, 107-117. [CrossRef]

15. Smith, K.M.; Eaton, A.D.; Finlayson, L.M.; Garside, P. Oral tolerance. Am. J. Respir. Crit. Care Med. 2000, 162, S175-S178. [CrossRef] [PubMed]

16. Weiner, H.L.; da Cunha, A.P.; Quintana, F.; Wu, H. Oral tolerance. Immunol. Rev. 2011, 241, 241-259. [CrossRef]

17. Da Silva, A.C.; Massa, S.; Sant'Anna, O.A. Preliminary results corroborating the polygenic control of immunological tolerance. Braz. J. Med. Biol. Res. 1990, 23, 581-584. [PubMed]

18. Da Silva, A.C.; de Souza, K.W.; Machado, R.C.; da Silva, M.F.; Sant'Anna, O.A. Genetics of immunological tolerance: I. Bidirectional selective breeding of mice for oral tolerance. Res. Immunol. 1998, 149, 151-161. [CrossRef]

19. Da Silva, M.F.; Nóbrega, A.; Ribeiro, R.C.; Levy, M.S.; Ribeiro, O.G.; Tambourgi, D.V.; Sant'Anna, D.A.; da Silva, A.C. Genetic selection for resistance or susceptibility to oral tolerance imparts correlation to both Immunoglobulin E level and mast cell number phenotypes with a profound impact on the atopic potential of the individual. Clin. Exp. Allergy 2006, 36, $1399-1407$. [CrossRef]

20. Wambre, E.; Jeong, D. Oral Tolerance Development and Maintenance. Immunol. Allergy Clin. N. Am. 2018, 38, 27-37. [CrossRef] [PubMed]

21. Brigatte, P.; Hoffmann, F.A.; Bernardi, M.M.; Giorgi, R.; Fernandes, I.; Takehara, H.A.; Barros, S.B.; Almeida, M.G.; Cury, Y. Tolerance to the antinociceptive effect of Crotalus durissus terrificus snake venom in mice is mediated by pharmacodynamic mechanisms. Toxicon 2001, 39, 1399-1410. [CrossRef] 
22. Chippaux, J.P. Incidence and mortality due to snakebite in the Americas. PLoS Negl. Trop. Dis. 2017, 11, e0005662. [CrossRef]

23. Queiroz, G.P.; Pessoa, L.A.; Portaro, F.C.; Furtado Mde, F.; Tambourgi, D.V. Interspecific variation in venom composition and toxicity of Brazilian snakes from Bothrops genus. Toxicon 2008, 52, 842-851. [CrossRef]

24. Giles, A.R. Guidelines for the use of animals in biomedical research. Thromb. Haemost. 1987, 58, 1078-1084. [CrossRef] [PubMed]

25. Bradford, M.M. A rapid and sensitive method for the quantitation of microgram quantities of protein utilizing the principle of protein-dye binding. Anal. Biochem. 1976, 72, 248-254. [CrossRef]

26. Mercuri, L.P.; Carvalho, L.V.; Lima, F.A.; Quayle, C.; Fantini, M.C.; Tanaka, G.S.; Cabrera, W.H.; Furtado, M.F.; Tambourgi, D.V.; Matos, J.d.R.; et al. Ordered mesoporous silica SBA-15: A new effective adjuvant to induce antibody response. Small 2006, 2, 254-256. [CrossRef] [PubMed] 